\title{
Das Institut für Plasma- und Lasertechnik der TFH Wildau
}

\author{
Harald Beyer, Andreas Harendt, Tobias Koschlig, Siegfried Rolle, Werner Roß, Hans Volkmann \\ Gewidmet H. Gründel †12.6.1996
}

\section{Eine kurze Historie}

Am 31.12.1996 endete das Wissenschaftler-IntegrationsProgramm (WIP) in den neuen Ländern. Es war angelegt worden, um Teilen der in der Akademie der Wissenschaften der DDR arbeitenden (und mehrfach positiv evaluierten) Wissenschaftlern eine Übergangsphase zu finanzieren, in der sie einerseits ihre Arbeit kontinuierlich fortsetzen konnten und andererseits die Integration in einer Hochschule erfolgen sollte, da die Forschungslandschaft der DDR an die bundesdeutschen Strukturen anzupassen waren. Das vorab auf zwei Jahre angelegte, 1992 gestartete Programm konnte bis 1996 verlängert werden, wenn es gelang, eine Hochschule zu finden, die bekundete, an einer Integration dieser Wissenschaftler in ihrer Einrichtung interessiert zu sein.

Ziellochschule der Wissenschaftler in der von Prof. Dr. sc. H. Gündel gegründeten Gruppe, die sich in Fortführung ihrer langjährigen Forschungstätigkeit auf dem Gebiet der nichtthermischen Plasmen (auch Niedertemperaturplasmen), insbesondere zur Erzeugung von Laserlicht beschäftigte und auch noch beschäftigt, war die TFH Wildau.

Dies war für eine Berliner Einrichtung einerseits ungewöhnlich, lag aber andererseits auch nahe: In der Gründungsphase der TFH Wildau lag ein besonderes Schwergewicht auf den modernen Technologien, was sich auch dadurch ausdrückte, daß es einen Fachbereich Physikalische Technik gab, der heute im Fachbereich Ingenieurund Wirtschaftsingenieurwesen als Studienrichtung weiter besteht. Da sich fuir die Zukunft eine immer breitere, auch ingenieurmäßige Anwendung der Plasmaund Lasertechnik abzeichnet und an den Deutschen Fachhochschulen dieses Gebiet (insbesondere die Plasmatechnik) kaum in der Lehre vertreten wird, wurde von beiden Seiten - der TFH Wildau auf der einen und der sich damals noch als WIP AG Niedertemperaturplasmen bezeichnenden Gruppe aus Berlin auf der anderen - eine Integration in einem Institut für Plasma- und Lasertechnik (IPLT) der TFH Wildau im Fachbereich Physikalische Technik angestrebt.

Bis zum 31.12.1996 war die Finanzierung der Gruppe aus den Mitteln des WIP gesichert. Darunter auch die räumliche Unterbringung der Lasertechnik und anderer Labore im Gebäude Berlin Hausvogteiplatz, dem ehemaligen Zentralinstitut für Elektronenphysik (ZIE) der AdW. (Die neu einzurichtende Plasmaspritztechnik ist von Anfang an auf dem Gelände der TFH in Wildau aufgebaut worden.)

Mitte 1996 zeichnete sich dann deutlich ab, daß eine Fortsetzung der Arbeit in den Berliner Räumen nicht möglich war. So kam es im Dezember 1996 zum Umzug fast des gesamten Gerätebestandes nach Wildau. Zum 1. April 1997 konnten dann die Wissenschaftler des IPLT ihre Arbeit fortsetzen bzw. mit der Wiedereinrichtung der Labore beginnen, da es ihnen und der TFH Wildau gelungen war, Förderung aus dem HSP III (Land und Bund jeweils zu $50 \%$ ) für zwei Jahre einzuwerben.

Mitte bis Ende 1997 waren die Labore wieder voll arbeitsfähig, so daß in diesem Jahr die Mitarbeiter des IPLT neben ihrer Forschungstätigkeit sowie der Technologieberatung auch wieder studentische Projekte, Praktika und Beiträge zur Ausbildung in der Studienrichtung Physikalische Technik in den Fächern Laser und Plasmatechnik anbieten können.

\section{Die Labore des IPLT}

\subsection{Das Laserlabor im Haus 3}

Im Laserlabor des IPLT stehen zu den verschiedensten Zwecken unterschiedliche leistungsfähige Laser zur Verfuigung:

\subsection{1 $\mathrm{CO}_{2}$-Bearbeitungslaser}

Bei diesem Laser handelt es sich um ein nicht kommerzielles Gerät aus eigener Entwicklung. Es zeichnet sich insbesondere dadurch aus, daß durch Variation der Betriebsparameter (wie Zusammensetzung des Lasergasgemisches und die Einstellung des Betriebsdruckes) der Laserimpuls in weiten Grenzen variiert werden kann: Die Impulsdauer läßt sich einstellen zwischen 10 und $200 \mu$ s bei Impulsenergien zwischen 200 und $500 \mathrm{~mJ}$ im transversalen Grundmode. Dies gestattet einerseits die Bearbeitung (insbesondere das Bohren und Schneiden) der verschiedensten, insbesondere auch nichtmetallischer Werkstoffe. Andererseits ermöglicht es die Untersuchung der Realisierbarkeit der Bearbeitung verschiedener Materialien mit solch einem Lasersystem. ${ }^{1-4}$

Die Antwort auf die Frage, ob sich eine bestimmtes Bearbeitungsergebnis mit einem Laser erzielen läßt, ist nicht trivial: Der eigentliche Materialbearbeitungsprozeß besteht aus einem komplexen Wechselspiel verschiedenster physikalischer Mechanismen. Beginnend mit der Absorption des Laserlichtes an der Werkstoffoberfläche (wenn man den Fokus des Laserstrahles nicht über die Oberfläche des Targets gelegt hat, und so unmittelbar ein Plasma erzeugt, wie z. B. bei den LPVDVerfahren) ${ }^{5}$, dem Aufschmelzen, Austreiben, Verdampfen und lonisieren des verdampften Materials bis hin zur Reflexion des Laserstrahls und damit der Rückkopplung auf das Lasersystem selbst. Damit dient dieser Arbeitsplatz unter anderem auch der Technologieberatung in der Region Berlin-Brandenburg. 
Das leistungsfähige Netzgerät und die im Lasersystem integrierte Gasumwälzung und -kühlung erlaubt den Betrieb bei einer Folgefrequenz bis zu $200 \mathrm{~Hz}$, was einer mittleren Leistung des Lasers von $1 \mathrm{~kW}$ entspricht. Da der Laserplatz außerdem mit einer Strahlformungsund Strahlfuihrungseinrichtung sowie einem computergesteuerten Kreuztisch, der eine Positioniergenauigkeit von $5 \mu \mathrm{m}$ besitzt, ausgestattet ist, können dort komplizierte Bearbeitungsaufgaben übernommen werden. Beispielhaft seinen hier die folgenden, durch das Laserlabor des IPLT erbrachten Dienstleitungen, angeführt:

- Für das Institut für Niedertemperatur-Plasmaphysik in Greifswald wurden in einer Kleinserie Titanringe mit Innendurchmessern im Bereich von 0,3 bis $1,6 \mathrm{~mm}$ geschnitten. Diese Ringe wurden als Lötringe zum Verschließen von sich in der Entwicklung befindlichen Speziallampen benötigt. Die Anfertigung verschiedener Stanzwerkzeuge wäre im Vergleich zum Laserschneiden bei den benötigten kleinen Stückzahlen wesentlich teurer gewesen.

- Für das Max Planck Institut für Plasmaphysik wurden aus extrem feinen Nickelmetallnetzen (Maschenweiten um die $10 \mu \mathrm{m}$ ) paßgerechte Formen geschnitten, die beim Aufbau bestimmter Plasmasonden benötigt wurden. Was die Präzision und den Erhalt der Formstabilität des Netzes im Fall dieser Sonderfertigung betrifft, hat sich das Laserschneiden als allen anderen Verfahren überlegen erwiesen.

\subsection{2 $\mathrm{CO}_{2}$-TEA Beschriftungslaser}

Ebenfalls als Prototyp einer Eigenentwicklung, die in Kleinserie für den industriellen Einsatz gebaut worden ist, steht im Laserlabor ein sogenannter $\mathrm{CO}_{2}$-Beschriftungslaser zur Verfuigung. Dieser Laser erzeugt großflächige und kurze Laserimpulse die durch eine Maske projiziert, über verschiedene Wirkmechanismen (z. B. Verdampfen von Lackschichten oder Oberflächenmodifikationen) das Beschriften von Massengütern in hocheffektiver Weise gestatten (man muß aufgrund der Kürze des Impulses das zu beschriftende Produkt nicht anhalten).

Der Laser ist zur Zeit nur für Ausbildungszwecke vorgesehen. Dabei ist beabsichtigt, die Studenten an dem oben beschriebenen Bearbeitungslaser eine Beschriftungsmaske erstellen zu lassen (Unikatfertigung) und verschiedene Materialien am Beschriftungslaser auf Tauglichkeit bezuiglich Laserbeschriftbarkeit hin zu untersuchen.

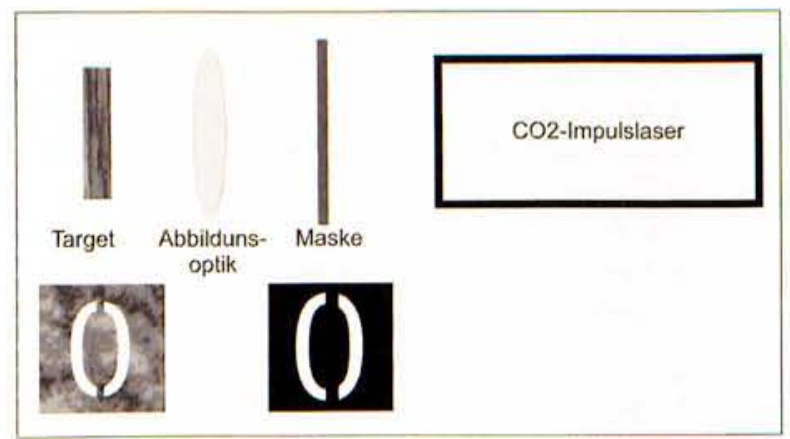

Abb. 1: Prinzip der Beschriftung mit einem $\mathrm{CO}_{2}$-Impulslaser: Befindet sich das Target in Beschriftungsposition, wird der Laserpuls ausgelöst. Charakteristisch bei der Laserbeschriftung sind die Schatten der Stege, welche die inneren Teile der Maske halten.

\subsubsection{Excimerlaser}

1996 konnte das IPLT einen kommerziellen Hochleistungslaser erwerben. Es handelt sich um einen $\mathrm{Kr} F$ Excimerlaser, der $500 \mathrm{~mJ}$ Impulsenergie bei $\lambda=248 \mathrm{~nm}$ und Impulsdauern im ns-Bereich erzeugt. Damit steht ein Laser zur Verfügung, der im UV Impulse mit Spitzenleistungen von einigen MW liefert.

Der Laser selbst ist einsatzbereit. Zur Anwendung in der Materialbearbeitung (respektive Materialbearbeitungsuntersuchungen) wird er gelangen, wenn eine entsprechende Strahlführungs- und Formungseinrichtung erworben werden kann. Der Strahl wird dabei auf dem bereits für den $\mathrm{CO}_{2}$-Bearbeitungslaser vorhandenen computergesteuerten Kreuztisch geführt. Damit wird ein großer Bereich von Materialien, die der Bearbeitung mit $\mathrm{CO}_{2}$-Lasern nicht oder nur sehr schwer zugänglich sind (im Infraroten hochreflektierende oder hochtransparente Substanzen) der Lasermaterialbearbeitung erschlossen. Es ist auch vorgesehen, diesen Laser für Polymerisation in dünner Schichten zu verwenden. Angedacht ist ebenfalls die Installation eines LPVD-Arbeitsplatzes. Im Rahmen der im IPLT bearbeiteten Forschungsthemen soll er als Quelle für laserinduzierte Fluoreszenz in spektroskopischen Untersuchungen eingesetzt werden.

\subsubsection{Argon-Ionenlaser}

Aus den Beständen des ZIE konnte das IPLT einen $\mathrm{Ar}^{+}$. Laser übernehmen. Es handelt sich um ein leistungsfähiges kommerzielles Gerät. Der Laser arbeitet auf verschiedenen Wellenlängen zwischen $\lambda=350-520 \mathrm{~nm}$. Im nichtselektiven Betrieb ${ }^{6}$ erreicht er eine cw-Gesamtleistung von $10 \mathrm{~W}$.

Ein vorhandener Laserscanner ${ }^{7}$ kann den Einsatz solcher Geräte in Gebieten wie der Unterhaltungsindustrie (Diskotheken etc.) demonstrieren. Aufgrund der Sichtbarkeit des von diesem Laser emittierten Lichtes ist allerdings vorgesehen, ihn auch in einem technischen Projekt mit Studenten in einem aufzubauenden Holografiemeßplatz einzusetzen.

Für die Zukunft sind Untersuchungen für den Einsatz bei der Oberflächenbearbeitung (z. B. Annealing für Halbleiter sowie Verglasung) vorgesehen.

Der vorhandene Laser ist in Hinblick auf besondere Effizienz bei der Anregung der UV Linien entwickelt worden: Damit ergibt sich auch prinzipiell die Möglichkeit zum Pumpen von Farbstofflasern und damit auch des Einsatzes in diagnostischen Methoden wie der Untersuchung von Plasmen und neutralen Gasen (Luft).

\subsubsection{Weitere Laser im Laserlabor und geplante Erweiterungen}

Neben den oben beschriebenen Lasern des Laserlabors sind noch vorhanden: Als Eigenentwicklung ein kleiner, abgeschmolzener Stickstofflaser $(\lambda=337 \mathrm{~nm})$. Er kann - auch über die Möglichkeit des Pumpens eines Farbstofflasers - für diagnostische Zwecke, z. B. für Kurzzeitinterferometrie und -fotografie, genutzt werden. Prinzipiell ist der $\mathrm{N}_{2}$-Laser auch für die Mikromaterialbearbeitung geeignet (ähnliche Parameter wie YAG-Laser) und wird seit 1978 (!) beim Entwurf von Halbleiterschaltkreisen industriell eingesetzt. Die dafür erforderlichen hochwertigen abberationsfreien UV- 
Optiken stehen allerdings im IPLT nicht zur Verfügung. $\mathrm{Zu}$ rein diagnostischen Zwecken steht ein (im P- und Q-Zweig der Rotationsbanden) durchstimmbarer $(\lambda=9,4-11,2 \mu \mathrm{m})$ kommerzieller $\mathrm{CO}_{2}$-Laser mit einer $\mathrm{cW}$ - Ausgangsleistung von etwa $3 \mathrm{~W}$ zur Verfügung. Angeschafft und bisher eingesetzt wurde er für Verstärkungsmessungen von mit Hochfrequenz angeregten $\mathrm{CO}_{2}$-Lasern.

Nach dem Umzug vom Hausvogteiplatz in Berlin nach Wildau ist ein vorhandener $\mathrm{cw}-\mathrm{CO}_{2}$-Laser der mittleren Leistungsklasse noch nicht wieder aufgebaut worden. Dies soll einem Projekt mit Studenten vorbehalten bleiben. Ein mit Hochfrequenz angeregter $\mathrm{CO}_{2}$-Laser $\left(\mathrm{CO}_{2}\right.$-slab-Laser), der im Ergebnis des vom BMFT geförderten Projekts Diffusionsgekühlte Schichtentladungen mit Hochfrequenz und Mikrowellenanregung für Hochleistungs- $\mathrm{CO}_{2}$-Laser (Förderkennzeichen $13 \mathrm{~N} \mathrm{5941)}$ entstand, kann als Labormuster zu Demonstrations- und Ausbildungszwecken (Praktikum Plasmaphysik: Hochfrequenzentladungen) im Laserlabor des IPLT ebenfalls wieder aufgebaut werden.

\subsection{Weitere Labore des IPLT im Haus 3}

Neben dem Laserlabor befinden sich im Haus 3 noch zwei weitere kleinere Laborräume: Das Plasmalabor und das Laserentwicklungs- und Elektroniklabor. In beiden Laboren werden die Forschungsthemen der durch das HSP III geförderten Wissenschaftler des IPLT bearbeitet.

\subsection{Die Plasmaspritzanlage}

\subsubsection{Beschichtungen für den Hochtechnologiebereich}

Die Beschichtungstechnologie mit ihren nahezu unbegrenzten Möglichkeiten der Veredlung von Substratoberflächen mit hochwertigen Funktionsschichten zählt mit zu den innovativsten Technologien. Die Neu- und Weiterentwicklungen auf dem Geräte-, Verfahrens- und Werkstoffsektor brachten eine Vielzahl neuer wirtschaftlicher Problemlösungen. Die Bedeutung dieser Technologie ist vor dem Hintergrund wirtschaftlicher Überlegungen zu sehen, denn Energieeinsparung und die Einsparung teurer Werkstoffe sind wesentliche Gesichtspunkte des Werkstoffverbundprinzips mit vielfältigen Aufgaben in der Reparatur, Instandhaltung und Neuteilfertigung in praktisch allen Industriebereichen.

Für die Realisierung von Verbundkonstruktionen stehen eine Vielzahl von Beschichtungsverfahren mit vielfältigen, den technischen und wirtschaftlichen Erfordernissen angepaßten Verfahrensvarianten zur Verfügung. Die Palette reicht von den konventionellen Plattierungs- und Auftragsschweißverfahren für häufig extreme Schichtdimensionen, den chemischen, elektrochemischen und thermochemischen Verfahren für vergleichsweise dünne Beschichtungen über die niederenergetischen thermischen Spritzverfahren des Lichtbogen- und Flammspritzens bis hin zu hochenergetischen Gasabscheidungsverfahren, Plasmaspritzverfahren und Hochgeschwindigkeitsspritzverfahren unter Nutzung reaktiver Gasmischungen. Die Beschichtungstechnologie stellt vielfach die einzige wirtschaftliche Alternative dar bzw. eröffnet durch die Eigenschaftskombination im Werkstoffverbund Möglichkeiten, die mit klassischem Kompaktwerkstoff- einsatz nicht realisierbar sind. Das gilt besonders für Hochtechnologiebereiche wie der Luft- und Raumfahrt, der Nukleartechnik, dem Turbinenbau, der Elektronik mit immer höheren Anforderungen an Funktionsschichten hinsichtlich elektronischer Eigenschaften sowie Verschleiß-, Gaskorrosions-, Oxidationsschutz und Wärmedämmungseigenschaften auch bei extremer Temperaturbelastung. Hochenergetische Beschichtungsverfahren, die diesen Aufgaben gerecht werden können, finden sich in den Verfahrensgruppen plasmagestützter Physical-Vapor-Deposition, Plasmaspritzen und Hochgeschwindigkeitsspritzen wieder.

\subsubsection{Die technische Ausstattung}

Die TFH Wildau bietet als einzige Hochschule im Raum Berlin-Brandenburg eine Spezialausbildung auf dem Gebiet der Plasmatechnik an. Mit der Zielstellung Studenten an modernsten Technologien auszubilden, der Technologieentwicklung für Firmen des Umlands, für Forschungsarbeiten und für den Technologietransfer wird eine robotergestützte Plasmaspritzanlage mit einer Nennleistung von $80 \mathrm{~kW}$ aufgebaut.

Die erforderlichen Peripherieeinrichtungen wie Luftfilter, Druckluftanlage, Sandstrahlanlage und Wärmetauscher wurden aus WIP-Mitteln finanziert bzw. durch zusätzliche Finanzmittel, die über Drittmitteleinwerbung verfuigbar gemacht werden konnten.

Der Steuerschrank der Plasmaspritzanlage wird mit Massenflußreglern nachgerüstet, so daß reproduzierbare Plasmaenthalpien einstellbar sind. Als Arbeitsgase können prinzipiell alle Gase verwendet werden, die keine übermäßige Elektrodenerosion bewirken, wobei die Gase Argon, Wasserstoff, Stickstoff und Helium gebräuchlich sind.

Die Anlage wird 1998 mit einem Industrieroboter, Verfahreinheit und Dreh-Kipp-Positionierer nachgerüstet, so daß auch komplex geformte Oberflächen reproduzierbar beschichtet werden können.

Damit steht im Raum Berlin-Brandenburg eine Technologie zur Verfügung, deren Bedeutung für alle Bereiche der Technik stark zunimmt. Der Einsatz dieses modernen Verfahrens wird in Klein- und Mittelständigen Unternehmen durch die vergleichsweise hohen Investitionskosten (ca. 700 TDM) bei im Einzelfall unzureichender Auslastung behindert. Deshalb soll die Plasmaspritzanlage sowohl für spezielle Technologieentwicklung als auch für die Auftragsfertigung verfügbar sein.

\section{Forschung im IPLT}

\subsection{Anwendungsorientierte Grundlagenforschung - ein Widerspruch?}

Üblicherweise wird die Forschung unterschieden in ${ }^{8}$ : Die Grundlagenforschung ..., die reine oder zweckfreie Forschung, die sich unabhängig von pragmatischen ${ }^{9}$ äußeren Zielorientierungen und Anwendungsbezügen um die Ausweitung des Erkenntnisstandes bemüht (sic!), und die angewandte oder Zweckforschung, die an der Lösung einzelner, ... praktischer Anliegen durch zielgerichtete Ausweitung und Anwendung von Forschungsergebnissen arbeitet.

Zwei Beispiele aus der Geschichte der Physik, die eng 
miteinander verflochten sind, mögen diese Unterscheidung zumindest diskutierbar machen: 10

Bis zum Jahr 1800, in dem J. Watt aus der mit M. Boulton 1775 gegründeten Dampfmaschinenfabrik ausschied, fand er viele Verbesserungen der von ihm 1765 erfundenen Niederdruckdampfmaschine. 1824, fünf Jahre nach dem Tod von J. Watt erschien als wichtigstes Werk des damals 29 Jahre alten N.L.S. Carnot (welches er während seiner Tätigkeit als Ingenieur von 1814-19 in der französischen Armee verfaßte) ${ }^{11}$. Er schreibt in der Einleitung: Trotz der mannigfaltigen Arbeiten über die Wärmemaschinen ... ist ihre Theorie doch sehr wenig fortgeschritten, und die Versuche zu ihrer Verbesserung sind fast nur vom Zufall geleitet. ${ }^{12}$ Carnot entwickelt dann die quantitativen Zusammenhänge des Kreisprozesses, findet die Beziehung für den thermodynamischen Wirkungsgrad und legte so (mit Clapeyrons ${ }^{13}$ 1834) die Grundlage eines der als gesichertsten geltenden Fundamente der Physik: Der Thermodynamik.

Carnots Forschung war also keineswegs zweckfrei und unabhängig von pragmatischen ... Zielorientierungen und Anwendungsbezügen, ganz im Gegenteil! Sie hat sich nicht um die Ausweitung des Erkenntnisstandes bemiiht, sie hat ihn ausgeweitet!

1854 erfand H. Goebel die Glühlampe. 1880 wurde in Menlo Park (Edisons Laboratorien bei New York) die Hochohmglühlampe erfunden, die eine Lebensdauer von rund 1000 Stunden hatte ${ }^{14}$.

Zu den Aufgaben der Physikalisch Technischen Reichsanstalt gehörte es unter anderem auch, elektrische Gluihlampen, die die Industrie einsandte, zu prüfen. Sowohl die Prüfung als auch die von der PTR geplanten systematischen Untersuchungen zur Verbesserung der Strahler (Gluihwendel, Gasgliihstrumpf) setzten Vergleichsnormale voraus und diese wiederum die Kenntnis des Strahlungsgesetzes.

M. Planck, der mit mehreren Physikern, die an der PTR solche Strahlungsmessungen (u. a. auch W. Wien) durchführten, persönliche Bekanntschaft hatte, begann 1895 seine keinesfalls zweckfreie Forschung zum Strahlungsgesetz. Planck ist dabei einen langen und mühevollen Weg gegangen und hat durch zielgerichtete Ausweitung und Anwendung von Forschungsergebnissen (insbesondere der Thermodynamik!!) in seinem Vortrag vor der Berliner Physkalischen Gesellschaft am 14.12.1900 die moderne Physik begründet. Der Autor will damit nicht behaupten, daß es nicht Forschung gibt und geben können muß, die unabhängig von nennbaren Zielstellungen agieren kann. Salopp gesagt: Viele große Entdeckungen, nicht nur in der Physik, sind dem unbändigen Spieltrieb von Genies zu verdanken. (Nichts weiter als Spiel - im positivsten Sinne des Wortes - oder vielleicht auch Kunst ist eine von pragmatischen ... Zielorientierungen freie Tätigkeit.)

Der Autor behauptet aber, daß ernsthaft betriebene Forschung, auch wenn sie pragmatische Zielstellungen hat, also auf einen nutzbringenden Zweck hin gerichtet ist, sich nicht nur um die Ausweitung des Erkenntnisstandes bemüht, sondern

1. durch die zielgerichtete Beschäftigung mit der Wissenschaft den Erhalt und die Ausweitung des Erkenntnisstandes begründet und
2. dabei immer öfter an Fragestellungen gerät, die im weitesten Sinne grundlegend sind.

Der letzte Punkt bedarf noch der Erläuterung: Die Grundgleichungen alleine der klassischen Physik, über deren Gültigkeit unter Physikern Konsens herrscht, sehen bestechend einfach aus: Die ... eindrucksvolle Schönheit der ... Maxwellschen Gleichungen ... veranlaßte Boltzmann zu dem Ausspruch: „, War es ein Gott, der diese Zeilen schrieb ...“.15 Das Phänomen der Ausbreitung elektromagnetischer Wellen ist mit diesen Zeilen ein für allemal und endgültig beschrieben. Da sie gegenuiber Lorentztransformation invariant sind, enthalten sie (ca. 40 Jahre vor Einsteins Formulierung) bereits die spezielle Relativitätstheorie. Die Fresnellschen Formeln können aus ihr abgeleitet werden: Sind sie eine Anwendung der Maxwellschen Gleichungen oder eine grundlegende Ableitung aus denselben?

In der üblichen Formulierung wird man Popow und Marconi zu den anwendungsorientierten Forschern zählen, denn sie hatten das Ziel, mit den Hertzschen Wellen Nachrichten zu übertragen und dabei ganz nebenbei die Grundlagen zumindest aber die Anfänge unserer medialen Welt geschaffen. Die Existenz elektromagnetischer Wellen ist eine fundamentale Aussage der Maxwellschen Theorie (1855-62). Waren die Hertzschen Versuche (1886-88) Grundlagenversuche, weil Hertz 1887 befragt, ob man mit seinen Wellen Signale übertragen könne, dies ausschloß, obwohl die ersten Versuche ja gerade darin bestanden, den erzeugten Funken in einem vom Erzeugungsort weit entfernten Detektor nachzuweisen.

1963 hatte E.N. Lorentz ${ }^{16}$ aus den hydrodynamischen Gleichungen ein vereinfachtes System von drei nichtlinearen Differentialgleichungen herausgeschält um atmosphärische Strömungsverhältnisse auf einem Computer zu simulieren. Diese Anwendung hat einen völlig neuen und nach Meinung des Autors das physikalische Weltbild verändernden Zweig der Physik hervorgebracht: Die nichtlineare Dynamik (populärer: Chaosphysik) ${ }^{17}$.

Der Autor betont noch einmal: Lorentz hatte die hydrodynamischen Gleichungen vereinfacht! Was wird die anwendungsorientierte Forschung bringen, wenn sich die experimentellen Möglichkeiten immer mehr erweitern eingeschlossen die höher werdende Rechnerleistung mit der die so simpel aufzuschreibenden Gleichungen der klassischen Physik immer tiefer zu analysieren sind? Wenn nun die Wissenschaftler im IPLT in ihrem Selbstverständnis davon ausgehen, anwendungsorientierte Grundlagenforschung zu betreiben, dann ist mit hoher Wahrscheinlichkeit davon auszugehen, daß keiner von ihnen einen neuen Zweig der Physik begründen oder grundlegende Erkenntnisse in dem Sinne liefern wird, daß die bekannten fundamentalen physikalischen Beziehungen auch nur korrigiert werden müßten. Die Projektbeschreibungen der Forschungsthemen, die im IPLT bearbeitet werden, zeigen aber, daß es sich bei der Plasma- und Lasertechnik um anwendungsorientierte Forschungsthemen handelt, die (bis auf die Hochenergiephysik und wenn wir von der Invarianz gegenüber Lorenztransformation bei den Maxwellschen Gleichungen 
absehen auch der Relativitätstheorie) zu ihrer Beschreibung de facto des ganzen zur Verfügung stehenden physikalischen Apparates bedürfen. Es ist also nicht verwunderlich, daß, wenn man sich auf diesem Gebiet mit beliebigen praktischen oder auch anwendungsorientiert genannten Fragen beschäftigt, man prinzipiell auf grundlegend $z u$ klärende Fragen stößt, wie es H. Gündel einmal ausdrückte. In diesem Sinn ist die hier getätigte Kritik an der geübten Trennung von grundlagen- und anwendungsorientierter Forschung zu verstehen und das Empfinden der Wissenschaftler im IPLT sich mit dem Betreiben anwendungsorientierter Grundlagenforschung in keinen Widerspruch zu begeben.

\subsection{Projekt: Laserlabor}

Das Laserlabor des IPLT versteht sich zu allererst als innovative Dienstleistungseinrichtung der anwendungsund grundlagenorientierten Forschung.

Für viele Standardanwendungen der Lasertechnologien, wie z. B. das makroskopische Schneiden oder Schweißen von Stahlblechen, gibt es heute fertige kommerzielle Lösungen, die je nach gewünschter Leistungsfähigkeit für mehr oder weniger Geld komplett mit dem dazugehörigen Handling von industriellen Anbietern aufgestellt werden.

Daneben gibt es einen großen Bedarf an innovativen Lösungen spezieller Bearbeitungswïnsche.

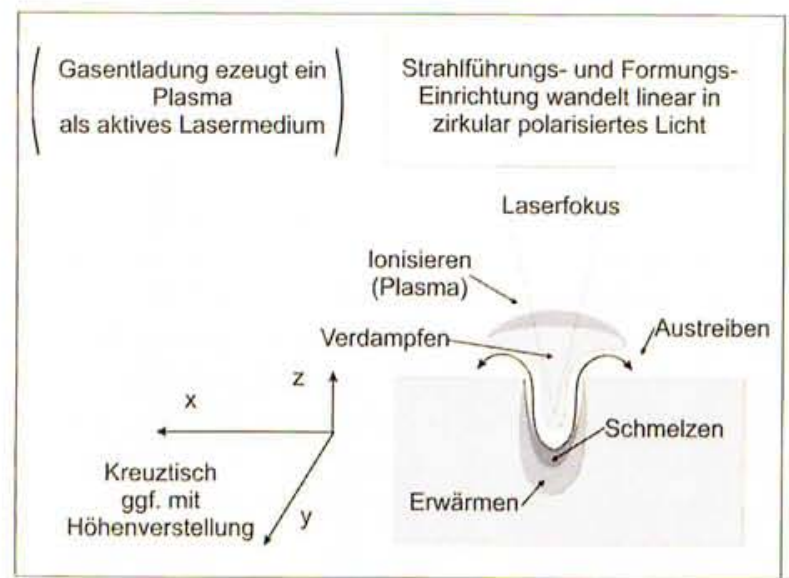

Abb. 2: Die wesentlichsten Komponenten einer Lasermaterialbearbeitung und die wichtigsten Prozesse bei der Bearbeitung.

Das Bearbeiten eines Materials mit einem Laserstrahl ist ein kompliziertes Wechselspiel verschiedener physikalischer Prozesse: Angefangen bei der einfachen Absorption des eingestrahlten Lichtes über die Erwärmung der Oberfläche bis zum Aufschmelzen und Verdampfen des Materials und der Ausbildung eines heißen, hochionisierten Plasmas an der Oberfläche des Targets kann man fortsetzen mit der Reflexion des vom Laser abgestrahlten Lichtes an der Oberfläche des Targets und der damit gegebenen Rückkopplung auf das Lasersystem selbst. Es ist also nicht möglich, von vornherein zu sagen, ob ein Material sich in einer gewünschten Weise mit einem bestimmten Laser modifizieren läßt oder nicht.

Die Arbeiten mit der Materialbearbeitungsanlage, die auf dem in weiten Parameterbereichen variierbaren $\mathrm{CO}_{2}$-TEA Laser basieren, haben sogar gezeigt: Vieles geht mit einem Typ $\mathrm{CO}_{2}$-Laser, mit einem anderen nicht. Soweit Unternehmen auch bereit sind, Lasertechnologien auf innovative Produkte anzuwenden, so werden sie kaum auf Verdacht eine (teure) Lasermaterialbearbeitungsanlage anschaffen. Ein Schwerpunkt in der Arbeit des Laserlabors im IPLT ist deshalb: Unterstiitzung von Unternehmen durch

- Bereitstellen von know how zum Einsatz von Lasertechnologie,

- Unterstïtzung und Realisierung experimenteller Forschungsmöglichkeiten zur Entscheidungsfindung bzgl. des Einsatzes von Lasertechnolgie zur Herstellung innovativer Produkte,

- Durchführung technologischer Entwicklungen, die im Ergebnis die Parametrisierung eines Lasersystems für eine spezielle Anwendung ergeben.

Lasertechnologien werden in Zukunft eine große Verbreitung finden. Es ist deshalb notwendig, in eine zeitgemäße und in die Zukunft orientierte Ausbildung von Ingenieuren technischer Fachrichtungen, die Lasertechnologien einzubeziehen. Damit ergibt sich der Schwerpunkt in der Arbeit des Laserlabors des IPLT an der TFH Wildau:

- Die zur Zeit im Laserlabor absolvierten Praktikumsversuche zur Materialbearbeitung mit $\mathrm{CO}_{2}$-Lasern sollen stufenweise ausgebaut werden, so daß auch nichtkonventionelle Lasertechnologien wie LPVD, Dünnschichtpolymerisation, Verglasen, Mikromaterialbearbeitung u.a. angeboten werden können.

Laser finden in den verschiedensten Verfahren der Meßtechnik einen breiten Einsatz. Deshalb soll das Laserlabor des IPLT

- in interdisziplinärer Weise durch den innovativen Einsatz entsprechend seinen Möglichkeiten die Forschung insbesondere an der TFH Wildau und im Land Brandenburg bei der Verwendung von Lasern in der Meßtechnik unterstuitzen.

In vielen Fällen der Fertigung lohnt der Einsatz von Lasertechnologien zur Herstellung innovativer Produkte, sei es für Kleinserien oder spezielle Einzelanfertigungen, die möglicherweise auch konventionell hergestellt werden könnten, dann aber teure Werkzeuge benötigen würden. Es gibt auch Fertigungsprobleme, die sich mit konventionellen Techniken überhaupt nicht bewältigen lassen. Die Kosten zur Anschaffung einer Lasermaterialbearbeitungsanlage sind allerdings sehr hoch und können - da eine Auslastung oft nicht gegeben ist - von kleineren Unternehmen oder auch Institutionen der Forschung nicht aufgebracht werden. Daraus ergibt sich eine weitere Tätigkeit des Laserlabors:

- Die vorhandenen Bearbeitungsanlagen für eine - vor allem auch wissenschaftlich betreute - wirtschaftliche Nutzung zur Herstellung von Spezialanfertigungen (auch in Kleinserien) für Unternehmen und Institutionen bereitzustellen. 


\subsection{Projekt: Wärmedämmschichten für Oberflächen- temperaturen $>1350^{\circ} \mathrm{C}$}

Im Rahmen eines Verbundprojektes im BMBF-Programm Neue Materialien für Schlüsseltechnologien des 21. Jahrhunderts-MaTech, sollen innovative Werkstoffsysteme entwickelt werden. Die Partner des bewilligten Verbundprojektes sind:

- SIEMENS AG

- BMW/ROLLS ROYCE AEROENGINES GmbH

- Deutsche Luft- und Raumfahrt e. V.

- Universität Erlangen-Nürnberg

- Universität Stuttgart.

Ziel des Verbundvorhabens ist die Entwicklung neuartiger Wärmdämmschichtmaterialien für heißgasbeaufschlagte Komponenten von Flug- und Stationärgasturbinen, die auch bei Oberflächentemperaturen $>1350^{\circ} \mathrm{C}$ zuverlässig einsetzbar sind. Grundvoraussetzung hierfür ist, daß neue Materialien nicht die bei den derzeit üblichen Zirconiumdioxid/Yttriumoxid-Systemen zu beobachtende Degradation der Materialeigenschaften zeigen.

\subsection{Projekt: Plasma zur Abgasreinigung}

Im IPLT wird untersucht, inwiefern sich nichtisotherme Plasmen zur Beseitigung toxischer gasförmiger Schadstoffe in Abgasen einsetzen lassen. Dies ist ein in der grundlagen- und anwendungsorientierten Forschung derzeit an verschiedenen Stellen bearbeitetes Gebiet. Der Versuch, Plasmen verschiedenster Art in der chemischen Synthese einzusetzen, ist nicht neu. Simpel gesagt, besteht das Problem also eigentlich nur darin, ein auf die Synthese zugeschnittenes Plasma zu schaffen: Es ist unstrittig, daß sich die Zahl möglicher chemischer Reaktionsprodukte eines in den Plasmazustand versetzten Reagenz gegenüber seinem sonst üblichen chemischen Gleichgewicht wesentlich erhöht. Diese einfache quantitative Erhöhung der Reaktionsmöglichkeiten sichert aber noch nicht die Möglichkeit, das chemische Gleichgewicht in eine bestimmte Richtung zu drängen. Das sich einstellende Reaktionsgleichgewicht hängt stark von der Art des erzeugten Plasmas ab. Erzeugt man mit Gasentladungen Plasmen, dann unterliegen die sich einstellenden Plasmaparameter, die von den verschiedensten Betriebsbedingungen abhängen, ihren eigenen Gesetzmäßigkeiten. Nur wenn es gelingt, Reaktoren zu bauen und Betriebsbedingungen zu finden, mit denen man Plasmen mit den zur Erzeugung einer bestimmten Reaktion benötigten Eigenschaften wenigstens angenähert erzeugen kann, ist die Plasmasynthese vieler (vielleicht sehr vieler) chemischer Substanzen wesentlich effektiver als herkömmlich (oder überhaupt erst) durchzuführen.

Die bisherigen Untersuchungen haben gezeigt, daß man einige, in Abgasen enthaltene gasförmige Schadstoffe, mit gezielt erzeugten Gasentladungen entweder zerstören oder in andere chemische (entweder anderweitig behandelbare oder unschädliche) Verbindungen umwandeln kann.

Ein Gegenstand der Arbeiten im IPLT ist die Identifizierung der wesentlichen Prozesse, die in nichtisothermen
Gasentladungen eine Zersetzung unerwünschter chemischer Verbindungen bewirken. Daraus können Erkenntnisse über die anzustrebenden Plasmaparameter in zukünftigen Plasmareaktoren gezogen werden.

Als Quellen für nichtisotherme Plasmen stehen dabei im IPLT folgende Reaktoren zur Verfuigung:

- Elektronenstrahl (100 keV, 1-4 W)

- Elektronenstrahlstabilisierte elektrische Entladung (bis $25 \mathrm{kV}$ über eine Entladungsstrecke von $22 \mathrm{~mm}$ )

- Impuls - Koronaentladung

- schnell geströmte Koronaentladung bei Atmosphärendruck

- Reaktor mit Impulsentladung bei ca. 0,1 atm

An diagnostischer Techniken ist vorhanden:

- FTIR-Spektrometer $\left(4000 \mathrm{~cm}^{-1}-500 \mathrm{~cm}^{-1}\right.$, Auflösung $1 \mathrm{~cm}^{-1}$ ) mit diversen Langweggasküvetten zum Nachweis von Molekülen im infraroten Spektralbereich

- MCP-CCD Spektrometer (180 nm-1000 nm) mit UV - White-Absorptionszelle. Durch eine hohe zeitliche Auflösung (10 ns) lassen sich kurzlebige Verbindungen diagnostizieren.

- hochwertige elektrische Meßtechniken (Oszillografen, Meßteiler, Stromwandler...)

Für weitere Diagnostiken steht ein Excimerlaser zur Verfuigung, der zum Pumpen eines Farbstofflasers geeignet ist.

\subsection{Projekt: Excimer-Laser mit gaußförmigem Strahlprofil}

Excimerlaser gehören aufgrund ihrer Strahlung im ultravioletten Spektralgebiet zu den am vielseitigsten eingesetzten Lasern in der Forschung und zunehmend auch in der industriellen Anwendung.

Alle am Markt erhältlichen Excimerlaser weisen jedoch, bedingt durch konstruktive Besonderheiten, eine unbefriedigende Strahlqualität auf. Diese Eigenschaft wirkt sich negativ auf die Fokussierbarkeit der Strahlung aus, wodurch der positive Einfluß der kurzen Wellenlängen z. T. wieder zunichte gemacht wird. Besonders störend ist dieser Effekt z. B. beim Bohren feinster Löcher oder beim Einkoppeln der Strahlung in Lichtleiter.

Das Ziel der Arbeiten besteht daher darin, einen Excimerlaser zu entwickeln, der die Vorteile der kurzen Wellenlänge mit einer guten Strahlqualität verbindet. Anzustreben ist ein kreisrunder Strahlquerschnitt mit Gauß-Profil. Die Laserentwicklung ist traditionelles Arbeitsgebiet von Mitarbeitern des IPLT, so daß die Erfahrungen genutzt werden, die unter anderem bei der Entwicklung von Stickstofflasern mit Gauß-ähnlichem Strahlprofil gewonnen wurden.

Die Strahlqualität eines jeden Gaslasers wird von der Geometrie des aktiven Mediums und den Eigenschaften des verwendeten Resonators bestimmt. In allen zur Zeit auf dem Markt erhältlichen Excimerlasern wird dieses aktive Medium durch eine schnelle Impulsentladung gebildet, die zwischen zwei einander gegenüberstehenden nahezu plattenförmigen Elektroden brennt. Damit hat das aktive Medium einen für eine gute Strahlqualität (kreisrunde Geometrie) ungünstigen rechteckförmigen Querschnitt, bei dem die Breite ein Vielfaches der Höhe 
beträgt. Diese Form wurde bisher durch die geforderten kurzen Stromimpulse und die daher notwendigen niedrigen Induktivitäten erzwungen. Zur Erzeugung kreisrunder Laserstrahlung sind verschiedene Wege möglich.

Im IPLT wurde der Weg über eine Entladungsanordnung gewählt, die von vornherein ein aktives Medium mit nahezu kreisrundem Querschnitt erzeugt. Es wird die Tatsache ausgenutzt, daß Entladungen, die zwischen zwei stiftförmigen Elektroden brennen, von vornherein eine Zylindersymmetrie aufweisen. Wegen der geforderten geringen Induktivitäten ist dabei die Länge der Entladung (Elektrodenabstand) auf wenige Zentimeter begrenzt, also zu klein, um eine ausreichende optische Verstärkung zu erreichen. Die Lösung besteht daher darin, elektrisch parallele Längsentladungen zwischen zwei Stiftelektroden optisch hintereinander anzuordnen. (vgl. Abb. 3)

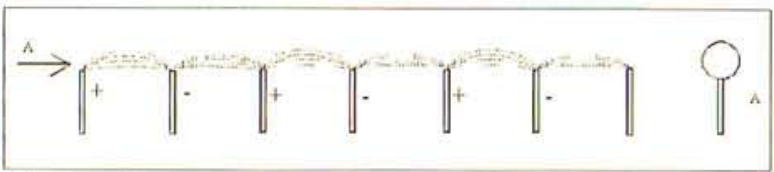

Abb. 3: Multieletroden-Längsanordmung

Dabei wechseln die Elektroden in ihrer Polarität. Jede Elektrode versorgt zwei benachbarte Elektrodenkanäle. Eine einfache Kopie dieser für Stickstofflaser eingesetzten Anordnung für Excimerlaser ist allerdings nicht möglich.

In $\mathrm{N}_{2}$-Laser werden Drücke von einigen 10 mbar verwendet, während Excimerlaser in Gasgemischen von mehreren bar betrieben werden müssen. Das macht $u$. a. den Einsatz einer Vorionisation erforderlich. Außerdem verlangen die aggressiven halogenhaltigen Excimer-Lasergase eine spezielle Gefäßtechnologie und ein spezielles Gashandling.

Der im Institut nach dem Prinzip des longitudinal angeregten Superstrahlers aufgebaute Excimerlaser hat ein Strahlprofil, die dem gaußförmigem ähnlich ist I. Die Anregung der Laserstrahlung ist jedoch von Impuls zu Impuls stark unterschiedlich. ${ }^{18}$

Ziel des Forschungsvorhabens ist es deshalb, die in dem vorhandene Laseraufbau als Hochspannungsschalter eingesetzte getriggerte Schaltfunkenstrecke durch ein Wasserstoff-Thyratron zu ersetzen. So werden sich vor allem gleichbleibende Schalteigenschaften und auch höhere Folgefrequenzen erreichen lassen.

Die „Puls zu Puls Stabilität" wird wesentlich von den Eigenschaften und der Geometrie der verwendeten Vorionisation bestimmt. Hier gilt es durch Optimierung der Parameter und des zeitlichen Einsetzens der bisher verwendeten Vorionisation ihre Effektivität und damit die Stabilität der Laserstrahlung zu verbessern. Insbesondere ist der Einsatz einer „stillen Entladung“ zur großvolumigen Vorionisation sowohl in Modellrechnungen als auch in der Praxis zu erkunden.

\subsection{Projekt: Theorie der Hochfrequenzentladung}

Sinnvolle physikalische Forschung erfordert immer Modellbildung. Gerade in der Gasentladungsphysik sind die Verhältnisse oftmals außerordentlich verwickelt, wobei über die physikalischen Grundlagen zur Beschreibung derselben Konsens herrscht:

Ausgangspunkt der Beschreibung einer Gasentladung ist die Boltzmannsche Fundamentalgeichung. Sie beschreibt die mikroskopische Änderung der Dichte und Geschwindigkeitsverteilungsfunktion jeder Sorte $i$ eines Gases bzw. der Spezies einer Gasentladung mit der Zeit:

$$
\frac{\partial(n f)_{i}}{\partial t}+v \nabla_{r}(n f)_{i}+\frac{F}{m_{i}} \nabla_{r}(n f)_{i}=\frac{\delta(n f)_{i}}{\delta t}
$$

Dabei ist die Quantenmechanik in der rechten Seite der Boltzmanngleichung, dem sogenannten Stoßterm, versteckt. Dieser beschreibt die elementaren Wechselwirkungen bei den Stoßprozessen, die auf jeden Fall die Geschwindigkeit (zumindest in der Richtung) der Stoßpartner und im unelastischen Fall auch noch Teilchen der einen Sorte in ein solches einer anderen Sorte überführen (z. B. Anregung oder Ionisation durch Elektronenstoß).

Da es sich bei dem zu untersuchenden Gegenstand um Gasentladungen handelt, kommen zu dieser äußerst schwierig zu handhabenden Gleichung noch die Maxwellschen Gleichungen der Elektrodynamik hinzu:

$$
\begin{array}{cc}
\operatorname{div} D=\rho & \operatorname{div} B=0 \\
\operatorname{rot} E=-\dot{B} & \operatorname{rot} H=\dot{D}+j \\
D=\varepsilon E & B=\mu H \\
j=-e \sum(n v)_{i}
\end{array}
$$

Gekoppelt wir dieses System mit der Boltzmannschen Fundamentalgleichung über die Kräfte, die auf die geladenen Teilchen wirken:

$$
F=e(E+v \times H)
$$

Damit hat man für die acht Größen (nf), $v, D, B, E, H, j$, $F^{19}$ acht Gleichungen zu deren Bestimmung (man berücksichtige, daß sich die Raumladung $\mathrm{r}$ aus den Dichten der Ladungsträger am Ort ergibt). Hinzu kommen noch zur Bestimmung der $n_{i}$ die Beziehungen der Gaskinetik, etwa

$$
p_{i}=n_{l} k T_{i}
$$

wobei $k T_{i}$ als ein Ausdruck für die mittlere kinetische Energie einer Spezies ist, die als Integral über die Geschwindigkeitsverteilungsfunktion $f_{i}$ zu finden ist. Dabei kann z. B. der Partialdruck einer jeden Sorte als Anfangsbedingung vorgegeben werden.

Damit ist prinzipiell eine in sich geschlossene selbstkonsistente Beschreibung einer Gasentladung aus den Grundgleichungen der Physik heraus möglich, aber bei dem derzeitigen Leistungsvermögen der Rechentechnik kaum realisierbar:

Glücklicherweise lassen sich aber in vielen praktisch interessierenden Fällen hinreichend vernünftige Näherungen dieses Systems vornehmen. Bei den im IPLT untersuchten Gasentladungen handelt es sich um Entladungen bei relativ hohem Druck ( $10 \mathrm{hPa}$ und darüber bis zu Atmosphärendruck). Untersucht man für solche Gase die Zeitabhängigkeit der Boltzmanngleichung, dann stellt man fest, daß sich über räumliche Bereiche, 
die der Größenordnung nach den mittleren freien Weglängen der Spezies entsprechen, in Zeiten, die den Stoßzeiten (der Zeit zwischen zwei Stößen) entsprechen, die Geschwindigkeitsverteilungsfunktionen den örtlichen Gegebenheiten, wie etwa dem lokal sich ausbildenden elektrischen Feld, anpaßt. Erwartet man also Lösungen, deren makroskopische Größen, wie mittlere Energie oder Raumladungsdichten sich nur in räumlichen Größenordnungen ändern, die ein vielfaches der mittleren freien Weglängen betragen und deren zeitliche Änderungen langsam im Vergleich zur Stoßfrequenz sind, dann kann man durch Bildung der Momente über die Boltzmanngleichung zu den Boltzmannschen Transportgleichungen übergehen. Man erhält dann die hydrodynamische Näherung der Beschreibung eines Plasmas bzw. einer Gasentladung in Form der Kontinuitätsgleichung, der Impuls- und Energiebilanzgleichung. Die Integrale über die Stoßterme ergeben dabei bestimmte Raten- bzw. Transportkoeffizienten, die der experimentellen Bestimmung durchaus zugänglich sind.

Im IPLT existiert eine langjährige, und anerkannte Erfahrung bei der hydrodynamischen Modellierung von Gasentladungen. Die jüngsten Anstrengungen auf diesem Gebiet waren der modellmäßigen Durchdringung des physikalischen Verständnisses von Hochfrequenzentladungen gewidmet ${ }^{20}$. Es besteht, aus den Ergebnissen dieser Untersuchungen heraus das Bedürfnis, dieses Modell quantitativ (Hinzunahme von Sorten) und qualitativ (Erweiterung auf ein zweidimensionales Modell) zu erweitern.

Aufgrund des Bedürfnisses insbesondere unverstandene Phänomene bei den Impulskoronaentladungen (aus dem Projekt Plasmen zur Abgasreinigung) verstehen zu lernen sowie der Notwendigkeit bei der Optimierung des Excimer-Lasers mit Gaußförmigem Strahlprofil zwecks Einstellen der Vorionisation ebenfalls Modellierungen vorzunehmen, ist damit begonnen worden, ein universelles Programmpaket zu entwickeln, welches es in Zukunft gestatten soll, hydrodynamische Modellierung in verschiedenen Geometrien unkompliziert durchfuihren zu können.

\section{Anmerkungen}

1 H. Beyer, W. Bohmeyer, W.Roß, W. Rudolph, $\mathrm{CO}_{2}$-Impulslaser in der Materialbearbeitung, Glas- und Keramikmagazin, Sprechsaal, Vol. 123 No.10, 1990, 1026

2 H. Beyer, W. Bohmeyer, A. Harendt, W. Roß, W. Rudolph, D. Wieseler, Laserbearbeitung mit einem $\mathrm{CO}_{2}$ - Impulslaser variabler Impulsdauer, 7. Tagung „Physik und Technik des Plasmas", Berlin 1990

3 W. Roß, H. Beyer, P. Bisin, A. Harendt, R. Rudolph, Erhöhung von Wirkungsgrad und Impulsdauer bei $\mathrm{CO}_{2}$-Impulslasern mit speziell angepaßten Anregungskreisen und Gasgemischen, Frühjahrstagung der DPG, Kiel 1991

4 H. Beyer, W. Roß, W. Rudolph, A. Michaelis, J. Uhlenbusch, W. Viöl, Interaction of $\mathrm{CO}_{2}$ laser pulses of microsecond duration with $\mathrm{Al}_{2} \mathrm{O}_{3}$ ceramic substrates, J. Appl. Phys. 70(1), 1991, 75-81

5 Laser Pulsed Vapor Deposition

6 Der $\mathrm{Ar}^{+}$-Laser schwingt in dieser Betriebsart auf mehreren Wellenlängen gleichzeitig an. Das Laserlicht ist dann nicht monochrom sondern aus mehreren Farben zusammengesetzt.

7 Das ist im Wesentlichen ein kleiner, beweglicher Spiegel, der über geeignete Software so geführt wird, daß sich der Laserstrahl mit hoher Geschwindigkeit auf eine Projektionsfläche lenken läßt und dort scheinbar geschlossene Linienzüge (Leuchtschriften) in hoher Variabilität entstehen.

8 Brockhaus Enzyklopädie, F. A. Brockhaus Mannheim, Bd. 7, 1988.

9 pragmatisch: |griech. pragmatikós „in Geschäften geschickt"] anwendungs-, sachbezogen, sächlich, auf Tatsachen beruhend. ( $\mathrm{Bd}$. 17)

$10 \mathrm{Zu}$ den historischen Angaben vergl.: W. Schreier, Biographien bedeutender Physiker, Volk und Wissen 1984

11 N.L.S. Carnot: Betrachtungen über die bewegende Kraft des Feuers und die zur Entwicklung dieser Kraft geeigneten Maschinen, Oswalds Klassiker der exakten Wissenschaften, Nr. 37, W. Engelmann, Leipzig 1892

12 ebenda, S. 6

13 Von diesem stammt auch die bekannte graphische Darstellung der Carnotschen Kreisprozesse.

14 Als Nebenprodukt entdeckte Edison dabei die Glühemission.

15 Zitiert aus Sommerfeld: Vorlesungen über theoretische Physik, Elektrodynamik, Leipzig 1964

16 E.N. Lorentz: Deterministic non periodic flow, J. Atmos. Sci., 20, 130-141 (1963)

17 Bereits Poincaré hatte sich mit der Frage der Empfindlichkeit der Lösungen bezüglich der Anfangsbedingungen bei klassischen mechanischen Systemen intensiv beschäftigt, und die Ljapunowsche Stabilitätstheorie ist ebenfalls seit etwa 100 Jahren bekannt. Der Verdienst von Lorentz u. a. besteht darin, die Jahrzehntelang geübte Reduzierung des Zufalls auf die quantenmechanische Unschärfe mikroskopischer Systeme beendet zu haben und das Bewußtsein für den deterministischen Zufall in der klassischen Physik neu geschärft zu haben.

18 H. Gündel, H. Volkmann: Excimer Laser mit Gaußförmigem Strahlprofil, Wissenschaftliche Beiträge der TFH Wildau 1/1996

19 In der Reihenfolge: Geschwindigkeitsverteilungsfunktion, Geschwindigkeit, elektrische Durchflutung, magnetischer Fluß, elektrische Feldstärke, elektrischer Strom, makroskopische Kraft.

20 A. Harendt: Some results from hydrodynamic modelling of diffusion cooled radiofrequency sheat discharges; Proc. ICPIG XXIII, Toulouse 1997

\section{Verfasser}

Prof. Dr. Siegfried Rolle

Dipl.-Phys. Harald Beyer

Dr.-Ing. Andreas Harendt

Dr. rer. nat. Tobias Koschlig

Dr. rer. nat. Werner Roß

Dipl.-Ing. Hans Volkmann

Technische Fachhochschule Wildau

Fachbereich Ingenieurwesen/Wirtschaftsingenieurwesen

Tel. (0 33 75) 507-145 